\title{
Ras and macropinocytosis: trick and treat
}

\author{
Cell Research (2013) 23:982-983. doi:10.1038/cr.2013.79; published online 18 June 2013
}

\begin{abstract}
Oncogene-driven adaptation of metabolism during tumorigenesis includes steps that stimulate the uptake of nutrients, especially glucose and glutamine, to sustain cell growth and proliferation. Macropinocytosis, a clathrin- and caveolin-independent endocytotic process that had previously been linked to the action of oncogenic Ras and Src, is now shown to contribute to amino acid uptake via enhanced delivery of extracellular proteins to lysosomes.
\end{abstract}

Tumorigenesis is a multistep process in which cells acquire the capacity to proliferate in an unrestrained fashion and ultimately to metastasize. The increase in cellular mass that normally precedes proliferation depends on the uptake of nutrients, used in anabolic processes to synthesize proteins, lipids and nucleotides. A large fraction of tumor cells generate ATP mostly from aerobic glycolysis with the concomitant production of lactate from pyruvate (the Warburg effect). Lipid synthesis requires cytoplasmic acetylCoA, which is generated from citrate exported from mitochondria. In addition, nucleotide synthesis requires aspartate from mitochondria. The diminished entrance of pyruvate into mitochondria in combination with the enhanced export of citrate and aspartate would result in a shortage of tricarboxylic acid (TCA) cycle and a consequent block in cell growth. Like other rapidly dividing cells, tumor cells compensate this decrease in TCA intermediates (anaplerosis) by glutaminolysis, in which glutamine is catabolized inside mitochondria to $\alpha$-ketoglutarate $(\alpha-K G)$ [1]. Glutamine can readily be taken up as an amino acid from plasma or interstitial fluid, but may also be derived from proteins following degradation in lysosomes.

Oncogenes like Ras and its downstream effector BRAF can stimulate nutrient uptake by upregulation or translocation of nutrient transporters to the plasma membrane [2]. Ras can also increase nutrient uptake by means of macropinocytosis via induction of membrane ruffles in a phosphatidylinositol-3 kinase (PI-3K)- and phospholipase Cdependent fashion [3, 4]. Macropinocytosis is a unique mode of endocytosis in which extracellular fluid is internalized in a clathrin- and caveolin-independent manner (reviewed in [5]) and that relies on actin-dependent ruffle formation. In a recent paper published in Nature, Commisso et al. [6] demonstrate that macropinocytosis occurs in tumor cell lines harboring an oncogenic Ras allele as well as in an in vivo model, where neoplastic pancreatic acinoductal cells were induced by tissue-specific expression of oncogenic Ras. This conclusion was reached by measuring uptake of FITC-labeled dextran and by inhibition by 5 -(N-ethyl-N-isopropyl)amiloride (EIPA). This latter compound is a relative specific inhibitor of macropinocytosis acting via lowering the cytoplasmic $\mathrm{pH}$, although it has also been reported to affect receptor-mediated endocytosis and to prevent acidification of early endosomes. The authors go on to show that supplying Ras-transformed cells with ${ }^{13} \mathrm{C}$-labeled yeast protein results in the appearance of fully labeled amino acids in the intracellular pool of amino acids. Furthermore, analysis of metabolites, such as $\alpha-K G$, fumarate and malate, confirmed that they contained
${ }^{13} \mathrm{C}$, demonstrating that amino acids derived from degraded yeast proteins are metabolized. The experiments with ${ }^{13} \mathrm{C}$ were performed in culture media with suboptimal glutamine concentrations. Interestingly, while cells in such media are hampered in their proliferation, this can be overcome by supplementing the culture media with BSA. Consistent with a role for macropinocytosis in proliferation, the rescue with BSA is EIPA sensitive, while rescue with glutamine or $\alpha-K G$ is not. A key question of course is whether macropinocytosis is important for tumor growth in vivo. The authors address this by using a heterotopic mouse model and show that tumors derived from pancreatic adenomacarcinoma cells with oncogenic Ras (Mia PaCa-2 cells) are sensitive to EIPA, whereas adenomacarcinoma cells without oncogenic Ras (BxPC-3 cells) result in EIPA-insensitive tumors. Taken together, these data suggest that inhibition of macropinocytosis may have therapeutic benefit for patients with tumors with activated alleles of Ras. As oncogenic Src can also induce macropinocytosis in a PI-3K-dependent manner, this may not be limited to tumors with activated Ras [3, 4].

With the growing interest in metabolic reprogramming of tumor cells, it has become clear that tumor cells are capable of selecting various metabolic settings that sustain proliferation [7]. For example, to increase their glycolytic flux, cells can employ various oncogene-dependent mechanisms, which include post-translational modification of enzymes involved in carbon metabolism [8] and gene transcription [9]. However, for most tumors it is currently 
unclear which of these mechanisms are operational and of therapeutic interest. Hence we are still far from a complete understanding of the interplay between oncogenes, tumor suppressors and key metabolic enzymes. The finding by Commisso et al. that EIPA has differential effects on pancreatic tumor cells in a heterotopic mouse model illustrates that pancreatic tumors can obtain their nutrients via distinct uptake systems. Furthermore, this study once again underscores that nutrient uptake does not only rely on nutrient transporters at the plasma membrane, but at least in certain cases the complete endosomal-lysosomal system has its contribution. It is interesting to note that the lysosome also functions as the site where the MTORC1 complex, a central regulator of growth and metabolism, is activated. mTORC1 activation at the cytoplasmic surface of lysosomes appears to be dependent on the presence of amino acids inside the lysosome [10]. As a consequence, Ras-induced macropinocytosis would be a means to simultaneously supply the cell with amino acids and allow for activation of mTORC1, which in turn stimulates anabolic processes (Figure 1). A further understanding of the control mechanism that couples the rate of lysosome maturation from endosomes and degradation inside lysosomes with the concentration of amino acids in the cytoplasm will enhance our understanding of tumorigenesis. It is conceivable that this will reveal novel roles for wellknown oncogenes as seen here for Ras.

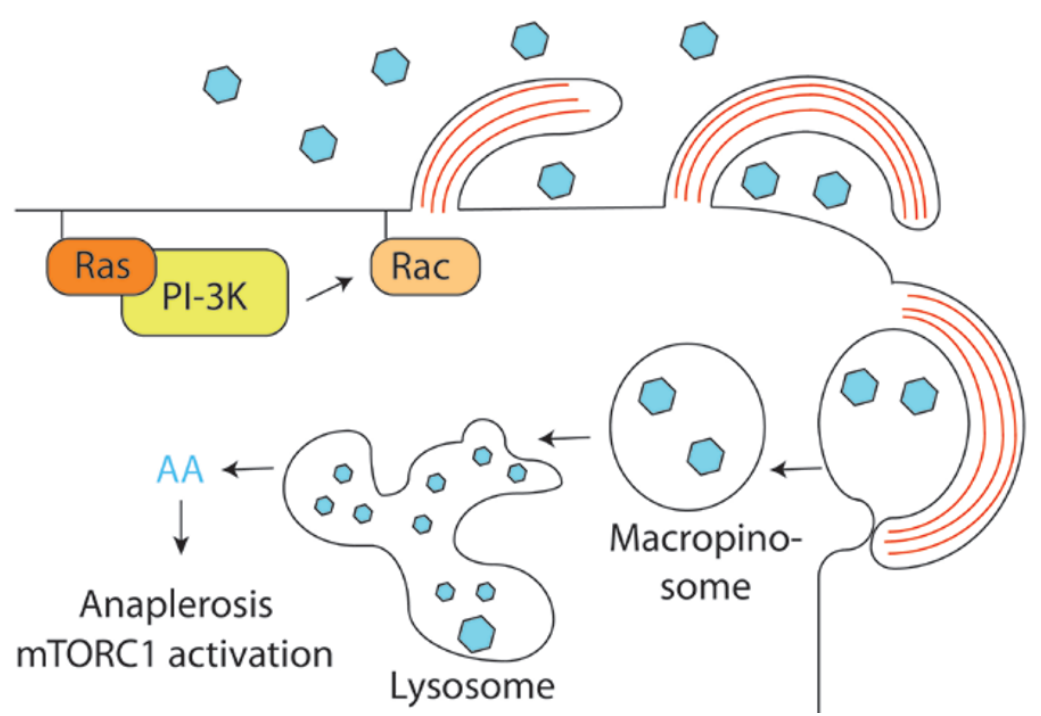

Figure 1 Schematic overview of Ras-induced macropinocytosis as a means of amino acid uptake. Ras induces actin-rich ruffles that fold back to induce macropinosomes. Fusion with the endosomal-lysosomal system and degradation of extracellular proteins yield amino acids that are transported from lysosomes and used to sustain cell growth and activate mTORC1. Actin filaments are indicated as red lines, extracellular protein and degradation products are depicted in blue. AA, amino acid.

Fried J T Zwartkruis ${ }^{1}$, Boudewijn M T Burgering ${ }^{1}$

${ }^{1}$ Molecular Cancer Research, Centre for Biomedical Genetics and Cancer Genomics Centre, University Medical Center Utrecht, Universiteitsweg 100, 3584 CG Utrecht, The Netherlands Correspondence: Fried J T Zwartkruis ${ }^{\mathrm{a}}$, Boudewijn M T Burgering ${ }^{b}$

aE-mail: G.J.T.Zwartkruis@umcutrecht.nl bE-mail: B.M.T.Burgering@umcutrecht.nl

\section{References}

1 Deberardinis RJ, Sayed N, Ditsworth D, et al. Curr Opin Genet Dev 2008; 18:54-61.

2 Yun J, Rago C, Cheong I, et al. Science
2009; 325:1555-1559.

3 Bar-Sagi D, Feramisco JR. Science 1986; 233:1061-1068.

4 Amyere M, Payrastre B, Krause U, et al. Mol Biol Cell 2000; 11:3453-3467.

5 Doherty GJ, McMahon HT. Аnпи Rev Biochem 2009; 78:857-902.

6 Commisso C, Davidson SM, SoydanerAzeloglu RG, et al. Nature 2013; 497:633637.

7 Ward PS, Thompson CB. Cancer Cell 2012; 21:297-308.

8 Anastasiou D, Poulogiannis G, Asara JM, et al. Science 2011; 334:1278-1283.

9 Duvel K, Yecies JL, Menon S, et al. Mol Cell 2010; 39:171-183.

10 Zoncu R, Bar-Peled L, Efeyan A, et al. Science 2011; 334:678-683. 